\section{Clinical genomic profiling of novel grey zone lymphoma paired lesions with sequential central nervous system involvement in two adolescent patients}

Grey zone lymphoma (GZL), defined as B-cell lymphoma, unclassifiable, with features intermediate between large B-cell lymphoma (LBCL) and classic Hodgkin lymphoma (cHL) (BCL-U-IND) is a rare diagnos- tic entity. ${ }^{1-3}$ Synchronous GZL, LBCL and cHL occurring simultaneously in the same patient, and sequential GZL, LBCL preceding or following a diagnosis of $\mathrm{CHL}$, are even less common. ${ }^{4}$ We identified two adolescent patients, a 17 year-old male (17M, case \#1) and 16 year-old female (16F, case \#2), who were diagnosed with stage IV nodular sclerosis cHL (NS-cHL) with primary mediastinal location and subsequent central nervous system (CNS) LBCL. Copy-number alterations were assessed using Affymetrix OncoScan ${ }^{\circledR}$ microarray analysis, and targeted next-gener-

Table 1. Clinicopathological summary of sequential grey zone lymphomas.

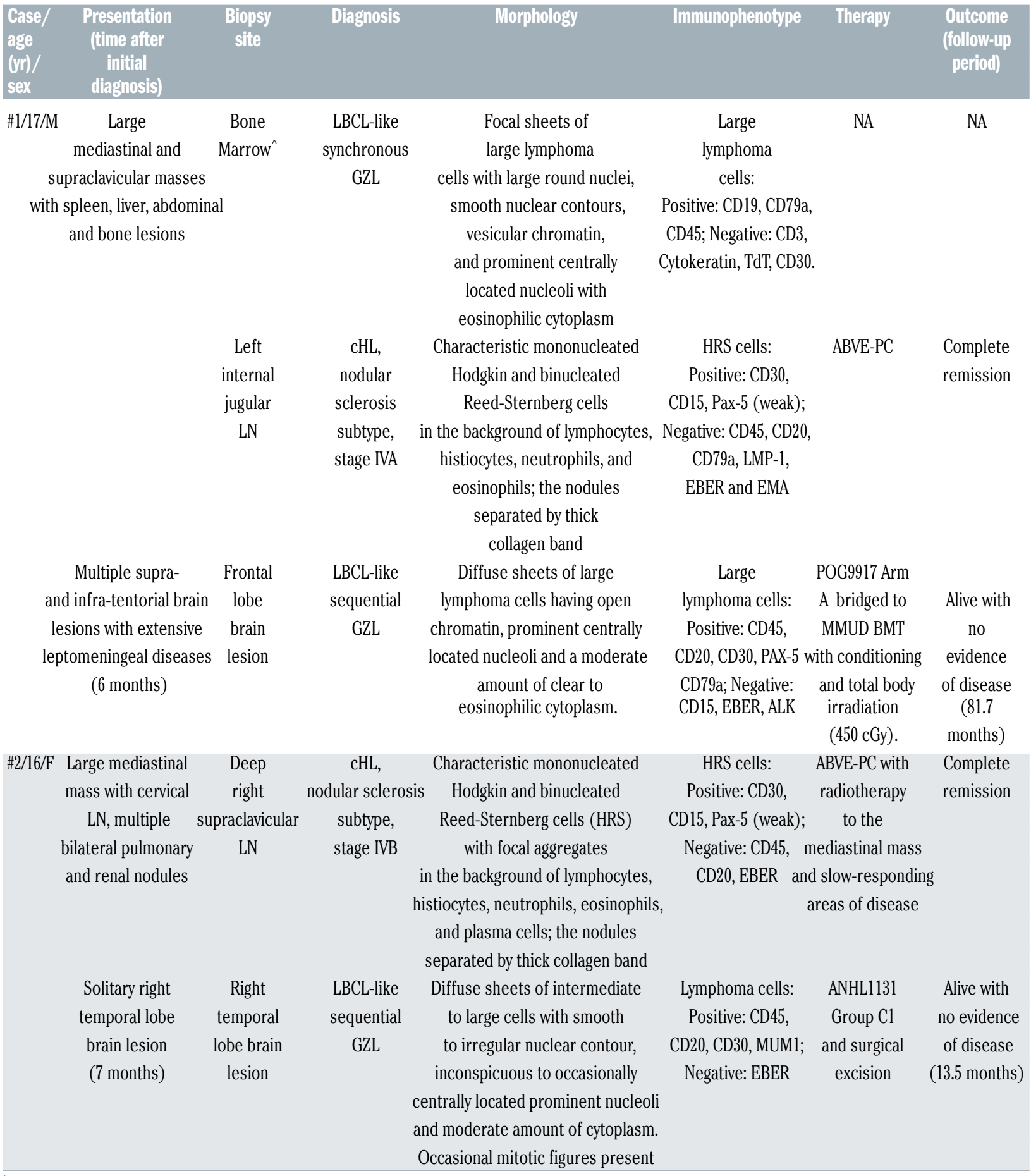

Outside bone marrow with limited slides reviewed as consultation. ABVE-PC: adriamycin, bleomycin, vincristine sulfate, etoposide phosphate, prednisone, cyclophos phamide; BMT: bone marrow transplant; cHL: classic Hodgkin lymphoma; F: female; GZL: grey zone lymphoma; HRS: Hodgkin and Reed-Sternberg; LBCL: large B-cell lymphoma; LN: lymph node; M: male; MMUD: mismatched unrelated donor; NA: not applicable. 
Table 2. Tissue-based cancer microarray and next-generation sequencing analysis of sequential grey zone lymphomas.

\begin{tabular}{|c|c|c|c|c|}
\hline \multirow{2}{*}{ Cytobands } & \multirow{2}{*}{ Size (Mbp) } & \multirow[b]{2}{*}{ Type } & \multicolumn{2}{|l|}{ Case \#1, 16M, Paired NS-cHL and CNS LBCL Microarray } \\
\hline & & & Array Nomenclature & Interpretation \\
\hline 2p16.3-p12 & 28.2 & Gain & $\operatorname{arr}[$ hg19] 2p16.3p12 (50,889,958-79,060,207)x3 & CNS LBCL only, Reported in GZL* \\
\hline $9 p 24.3-q 34.3$ & 140.9 & Gain & $\operatorname{arr}[$ hg19] 9p24.3q34.3(204,737-141,054,761)x3 & CNS LBCL only, Reported in GZL* \\
\hline $4 q 21.22-q 21.23$ & 1.1 & Loss & $\operatorname{arr}[$ hg19] 4q21.22q21.23(83,278,777-84,335,477)x1 & CNS LBCL only \\
\hline 6p25.3-p11.2 & 57.0 & $\mathrm{CN}-\mathrm{LOH}$ & $\operatorname{arr}[\mathrm{hg} 19]$ 6p25.3p11.2(204,908-57,160,035)x2 hmz & CNS LBCL only* \\
\hline 12p13.33-q24.33 & 133.6 & Gain & $\operatorname{arr}[$ hg19] 12p13.33q24.33(189,399-133,818,115)x3 & CNS LBCL only* \\
\hline Xp22.33-q28 & 155.0 & Gain & $\operatorname{arr}[$ hg19] Xp22.33q28(177,941-155,219,364)x2 & CNS LBCL only \\
\hline \multirow[t]{2}{*}{ Yp11.31-q11.23 } & 26.1 & Loss & $\operatorname{arr}[$ hg19] Yp11.31q11.23 $(2,660,162-28,799,935) \times 0$ & CNS LBCL only \\
\hline & & & Case \#1, 16M, Paired NS-CHL and CNS LBCL NGS & \\
\hline Gene & Pos (hg19) & RefSeq RNA & CDS; Protein; VAF (NS-cHL/LBCL) & Interpretation \\
\hline$A P C$ & chr5:112102044 & NM_000038.5 & c.157G >A; p.Gly53Arg; 0.05 & III, CNS LBCL only \\
\hline FAT4 & chr4:126239848 & NM_024582.4 & c.2282T>G; p.Leu761Trp; 0.37 & III, CNS LBCL only \\
\hline NOTCH3 & 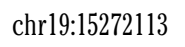 & NM_000435.2 & c.6326G >A; p.Arg2109Gln; 0.48 & III, CNS LBCL only \\
\hline CREBBP & $\operatorname{chr16:3819314~}$ & NM_004380.2 & c.2921C>A; p.Thr974Asn; 0.44/0.47 & III, Shared, Reported in GZL \\
\hline$A P C$ & chr5:112175211 & NM_000038.5 & c.3920T>A; p.Ile1307Lys; 0.56/0.45 & III, Shared \\
\hline ORAII & chr12:122064705 & NM_032790.3 & c.58G >A; p.Gly20Ser; 0.62/0.29 & III, Shared \\
\hline SETX & $\operatorname{chr} 9: 135145055$ & NM_015046.5 & c.7234A>G; p.Ile2412Val; $0.47 / 0.28$ & III, Shared \\
\hline SOS1 & chr2:39251255 & NM_005633.3 & c.1098T>A; p.Asp366Glu; 0.44/0.48 & III, Shared \\
\hline SYNE1 & chr6:152651971 & NM_182961.3 & c.13849A>C; p.Asn4617His; 0.41/0.47 & III, Shared \\
\hline SYNE1 & chr6:152630998 & NM_182961.3 & c.17174C>A; p.Thr5725Asn; 0.42/0.51 & III, Shared \\
\hline SYNE1 & chr6:152565729 & NM_182961.3 & c.19635G >T; p.Arg6545Ser; 0.44/0.46 & III, Shared \\
\hline \multirow[t]{2}{*}{ SYNE1 } & chr6:152464786 & NM_182961.3 & c.25091C>T; p.Pro8364Leu; 0.47/0.51 & III, Shared \\
\hline & & & Case \#2, 17F, Paired NS-cHIL and CNS LBCL. Microarray & \\
\hline Cytobands & Size (Mbp) & Type & Array Nomenclature & Interpretation \\
\hline $2 \mathrm{p} 25.3-\mathrm{q} 37.3$ & 243.0 & Gain & $\operatorname{arr}[$ hg19] 2p25.3q37.3(21,493-243,052,331)x3 & CNS LBCL only, Reported in GZL* \\
\hline $9 \mathrm{p} 24.3-\mathrm{q} 34.3$ & 140.9 & Gain & $\operatorname{arr}[\mathrm{hg19}]$ 9p24.3q34.3(204,737-141,054,761)x5 & CNS LBCL only, Reported in GZL* \\
\hline 16p13.13-p11.1 & 35.1 & Gain & $\operatorname{arr}[$ hg19] 16p13.13p11.1(83,886-35,271,725)x3 & CNS LBCL only, Reported in GZL \\
\hline 1p36.11-p35.3 & 3.0 & $\mathrm{CN}-\mathrm{LOH}$ & $\operatorname{arr}[\mathrm{hg19]}$ 1p36.11p35.3(25,194,298-28,160,199)x2 hmz & Shared \\
\hline 5p15.33-q23.3 & 128.0 & Gain & $\operatorname{arr}[$ hg19] 5p15.33q23.3(38,138-128,042,790)x3 & CNS LBCL only \\
\hline 5q23.3-q31.1 & 4.0 & Gain & $\operatorname{arr}[$ hg19] 5q23.3q31.1(128,063,275-132,042,740)x5 & CNS LBCL only \\
\hline $5 q 31.1-q 35.3$ & 49.2 & $\mathrm{CN}-\mathrm{LOH}$ & $\operatorname{arr}[\mathrm{hg19}]$ 5q31.1q35.3(131,530,440-180,698,312)x2 hmz & CNS LBCL only \\
\hline 6p25.3-p23 & 14.6 & CG-LOH & $\operatorname{arr}[$ hg19] 6p25.3p23(204,908-14,823,522)x3 hmz & CNS LBCL only* \\
\hline 6p23-p21.1 & 28.1 & $\mathrm{CN}-\mathrm{LOH}$ & $\operatorname{arr}[\mathrm{hg} 19]$ 6p23p21.1(14,984,113-43,101,670)x2 hmz & CNS LBCL only \\
\hline 12p12.3-q24.33 & 117.3 & Gain & arr[hg19] 12p12.3q24.33(16,480,948-133,818,115)x3 & CNS LBCL only* \\
\hline 15q11.1-q14 & 16.9 & Gain & $\operatorname{arr}[$ hg19] 15q11.1q14(20,161,371-37,079,572)x3 & CNS LBCL only \\
\hline $15 q 14-q 21.2$ & 12.5 & Loss & $\operatorname{arr}[$ hg19] 15q14q21.2(37,094,935-49,619,400)x1 & CNS LBCL only \\
\hline $15 q 21.2-q 26.3$ & 52.8 & Gain & $\operatorname{arr}[$ hg19] 15q21.2q26.3(49,643,377-102,397,317)x3 & CNS LBCL only \\
\hline $16 q 11.2-q 24.3$ & 43.7 & CN-LOH & 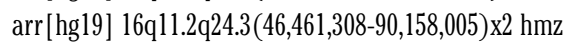 & CNS LBCL only \\
\hline 19p13.3-p13.3 & 1.1 & Gain & $\operatorname{arr}[$ hg19] 19p13.3(247,231-1,351,916)x3 & CNS LBCL only \\
\hline 21p11.2-q22.3 & 38.4 & Gain & $\operatorname{arr}[\mathrm{hg} 19]$ 21p11.2q22.3(9,648,314-48,097,610)x3 & CNS LBCL only \\
\hline 22q13.2-q13.33 & 7.7 & Loss & $\operatorname{arr}[$ hg19] 22q13.2q13.33(43,487,259-51,213,826)xl & CNS LBCL only \\
\hline Xp22.33-p22.11 & 22.3 & Loss & $\operatorname{arr}[\mathrm{hg} 19]$ Xp22.33p22.11(177,941-22,471,996)x1 & CNS LBCL onl y \\
\hline Xp22.11-q23 & 88.1 & $\mathrm{CN}-\mathrm{LOH}$ & $\operatorname{arr}[\mathrm{hg} 19]$ Xp22.11q23(22,634,971-110,738,270)x2 hmz & CNS LBCL only \\
\hline \multirow[t]{2}{*}{ Xq23-q28 } & 44.5 & CG-LOH & $\operatorname{arr}[\mathrm{hg} 19]$ Xq23q28(110,762,820-155,219,364)x3 hmz & CNS LBCL only \\
\hline & & & Case \#2, 17F, Paired NS-cHL and CNS LBCL NGS & \\
\hline Gene & Pos (hg19) & RefSeqRNA & CDS; Protein; VAF (NS-cHL/LBCL) & Interpretation \\
\hline TP53 & $\operatorname{chr17:7577569}$ & NM_000546.5 & c.712T>C; p.Cys238Arg; 0.51 & I/II, CNS LBCL only, Reported in GZL \\
\hline$F B X W 7$ & chr4:153249384 & NM_033632.3 & c.1394G>A; p.Arg465His; 0.45 & I/II, CNS LBCL only \\
\hline$C B L$ & $\operatorname{chr11:119170426}$ & NM_005188.3 & c.2656G >A; p.Glu886Lys; 0.40 & III, CNS LBCL only \\
\hline FAT1 & chr4:187557893 & NM_005245.3 & c.3818A>T; p.His1273Leu; 0.45 & III, CNS LBCL only \\
\hline KMT2D & chr12:49423015 & NM_003482.3 & c.14080G >C; p.Glu4694GIn; 0.50/0.30 & III, Shared, Reported in GZL \\
\hline RELN & chr7:103338388 & NM_005045.3 & c.1055A>G; p.Asn352Ser; 0.51/0.49 & III, Shared, Reported in GZL \\
\hline$E R G$ & chr21:39795460 & NM_001136154.1 & c.281T>G; p.Val94Gly; 0.46/0.33 & III, Shared \\
\hline ZFHX3 & chr16:72830889 & NM_006885.3 & c.5692G>C; p.Gly1898Arg; 0.47/0.95 & III, Shared \\
\hline
\end{tabular}

*Detected in both CNS LBCL cases. NS-cHL: classic Hodgkin lymphoma, nodular-sclerosing subtype; CNS: central nervous system; LBCL: large B-cell lymphoma; NGS: next-generation sequencing; Pos: genomic coordinate; RefSeq: reference transcript ID; CDS: coding sequence;VAF: variant allele frequency; Mbp: mega basepairs; GZL: grey zone lymphoma; CG-LOH: copy-gain loss of heterozygozity; cHL: CN-LOH, copy-neutral loss of heterozygosity. 


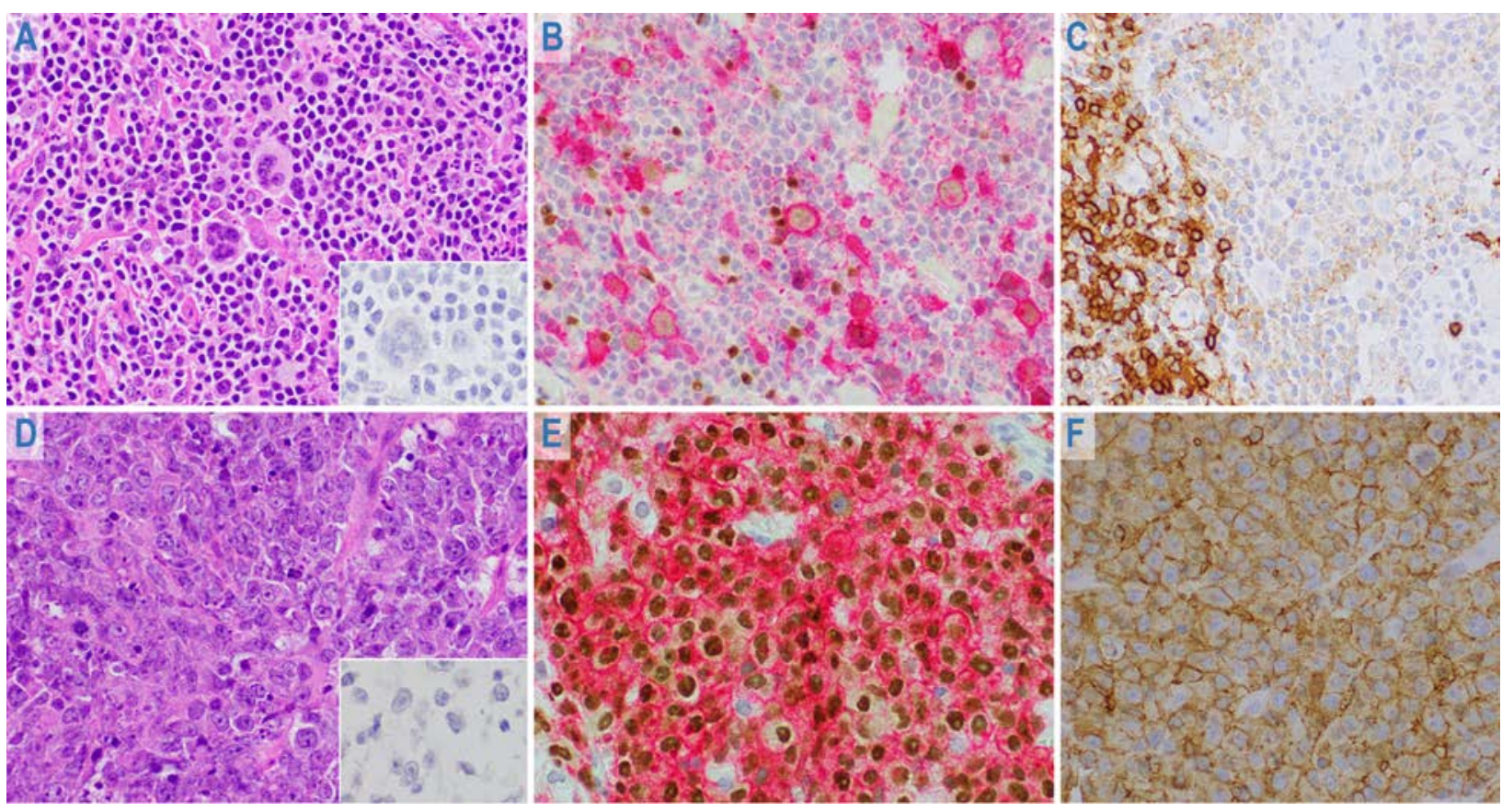

Figure 1. Representative pathologic findings of sequential grey zone lymphomas (Case \#1). Initial cervical lymph node biopsy shows classic Hodgkin lymphoma (upper panel, A to C). (A) Characteristic Hodgkin and Reed-Sternberg (HRS) cells are present in a polymorphous inflammatory background (hematoxylin and eosin stain[H\&E]); the HRS cells are negative for EBER (inset A). (B) The neoplastic HRS cells are positive for CD30 (red, membranous) and weekly positive for PAX-5 (brown, nuclear). (C) They are negative for CD20. (D to F) Lesional brain biopsy shows sequential central nevous system large B-cell lymphoma (lower panel). (D) Diffuse sheets of large lymphoma cells shows centrally located prominent nucleoli (H\&E); they are negative for EBER (inset D). (E) The lymphoma cells are diffusely positive for CD30 (red, membranous) with strong nuclear PAX-5 (brown) expression, (F) and express strong and homogeneous CD20.

ation sequencing (NGS) using a capture-based 152 gene custom-designed hematologic malignancy panel was performed on paired cHL and CNS LBCL tumors to assess for genomic alterations as previously described. ${ }^{5}$ These studies were performed under institutional-approved study protocols. We present the clinicopathologic and genomic features of the paired lesions in this previously unreported presentation of pediatric sequential GZL.

Case \#1, 17M. A 17 year-old male presented with large mediastinal and supraclavicular masses with disseminated spleen, liver, and bone lesions. Left cervical lymph node sampling revealed the classic histology and immunophenotype of NS-cHL (Figure 1A to C, Table 1). A concomitant outside bone marrow sample performed approximately 3 weeks prior revealed LBCL with sizable clusters of large lymphoma cells, consistent with a diagnosis of synchronous GZL. Staging bone marrow was negative for involvement by lymphoma. Complete remission was achieved after initial treatment with ABVE-PC chemotherapy regimen. Six months, after initial diagnosis ( 2 months post-therapy), several supra- and infra-tentorial brain lesions and extensive leptomeningeal disease appeared. A biopsy of a CNS lesion revealed diffuse sheets of large lymphoma cells having open chromatin, prominent centrally located nucleoli, and a moderate amount of clear to eosinophilic cytoplasm. The lymphoma cells showed diffuse and strong expression for CD45, CD20, PAX-5, CD30, and expression of CD79a, and were negative for CD15, EBER, and ALK (Figure 1D to F, Table 1). A diagnosis of BCL-U-IND consistent with sequential GZL was rendered. He was treated according to POG9917 Arm A as a bridge to bone marrow transplant with a mismatched unrelated donor and received total body irradiation (450 cGy). He was alive at 81.7 months follow-up.
Case \#2, 16F. A 16 year-old female presented with a large mediastinal mass with cervical lymphadenopathy and multiple bilateral renal and pulmonary nodules. NScHL was diagnosed from cervical lymph node biopsy; staging bone marrow was negative. She achieved complete remission after ABVE-PC and radiotherapy to the mediastinal mass and other slow-responding areas of disease. Seven months after initial diagnosis ( 2 months posttherapy), a solitary right temporal lesion was identified. A biopsy revealed essentially similar morphologic and immunophenotypic findings to the CNS lesion of case \#1 (Table 1), and a diagnosis of BCL-U-IND, consistent with sequential GZL was rendered. She was treated with ANHL1131 Group C1 and surgical excision and was alive at 13.5 months follow-up.

Molecular findings. The microarray and NGS results are summarized in Table 2. In both NS-cHL, near-diploid male or female genomes and no variants of established or potential clinical significance (Tier I/II, Table 2) were detected consistent with "negative" genomic profiles reported in bulk cHL lesions without Reed-Sternberg cell enrichment. ${ }^{6,7}$ In case \#2, a shared 3.0 MB region of copyneutral loss of heterozygosity (LOH) in chromosome 1p36.11-p35.3 was observed that was most likely germline in origin. Both CNS LBCL harbored complex cytogenomic arrays including 2 p16.1 and 9p24.1 gains (detected in both cases, Table 2, denoted by ${ }^{*}$ ) and 16 p13.3 copy-number abnormalities (case \#2 only). LOH of chromosome $6 p$ and gain of chromosome $12 p$ were also observed in both CNS LBCL (Table 2, denoted by *). NGS revealed shared NS-cHL/CNS LBCL variants of uncertain significance (VUS, Tier III) in CREBBP p.T974N (case \#1) and RELN p.N352S and KMT2D p.E4694Q (case \#2). The sequential CNS LBCL in case \#1 harbored addi- 
tional Tier III variants including APC p.G53R, FAT1 p.L761W, and NOTCH3 p.R2109Q. The sequential CNS LBCL in case \#2 harbored pathogenic (Tier I/II) TP53 p.C238R and FBXW7 p.R465H missense variants.

In this report, we detailed the clinicopathologic and molecular features of two adolescent patients with sequential GZL involving the CNS. Notably, this is the first report describing CNS involvement as a manifestation of sequential GZL, a finding which expands the clinicopathologic spectrum of this rare pediatric disease. Consistent with previous reports, both patients presented with mediastinal NS-cHL and advanced extranodal disease with similar histopathologic and immunophenotypic findings, and developed GZL in a similar chronologic fashion. ${ }^{4,8}$ The sequential CNS lesions showed differing morphologic and immunohistochemical profiles with strong and diffuse expression of several B-cell markers and CD30, the latter arguing against an extramediastinal primary mediastinal B-cell lymphoma (PMBCL) diagnosis, and the NS-cHL diagnosis preceded the diagnosis of LBCL temporally establishing the sequential GZL diagnosis. Additionally, the findings of synchronous GZL with subsequent development of sequential GZL in the first patient is also exceptional. Furthermore, unlike previous reports, an early evolution (e.g., second lymphoma diagnosis within 1 year) may not necessarily portend a poor clinical outcome $e^{4}$ given the favorable clinical responses in our two patients and a relatively long term follow-up in the first.

Recent molecular characterization of GZL supports the classification of two distinct subtypes of GZL: a "thymic" subtype that occurs in the anterior mediastinum and resembles Epstein-Barr virus (EBV)-negative $\mathrm{cHL}$ and PMBCL, and a "non-thymic" subtype which occurs outside the thymus and harbors TP53 mutations in a subset of cases., ${ }^{9,10}$ In our two patients, the CNS location and mutations in TP53 (case \#2) and other associated genes (e.g., CREBBP, RELN, and KMT2D) support a "nonthymic" GZL classification. The presence of complex genomic profiles is also consistent with dysregulated TP53 signaling, and both CNS LBCL harbored complex cytogenomic arrays with copy number abnormalities previously reported in GZL $\mathrm{L}^{11-13}$ and frequently reported in cHL and PMBCL. ${ }^{14,15}$ We acknowledge that a thorough investigation of enriched Reed-Sternberg cells from the $\mathrm{cHL}$ lesions and specific subsets of lesional cells may yield valuable molecular insights but this was beyond the scope of the current study.

In summary, we present the first report of sequential GZL with CNS involvement in two adolescent patients, and the first clinical genomic profiling of such paired lesions. These lesions showed chromosome aberrations identified in GZLs and NGS mutations associated with non-thymic GZL. These findings expand the clinicopathologic and genomic spectrum of this rare pediatric disease.

Cagla Yasa-Benkli, ${ }^{1}$ Andrea N. Marcogliese, ${ }^{1,2}$ Jennifer E. Agrusa, Adekunle M. Adesina, ${ }^{1,2}$ Howard L. Weiner, ${ }^{3}$ Kevin E. Fisher" and Choladda V. Curry ${ }^{\text {1\# }}$

${ }^{\#} K E F$ and $C V C$ contributed equally as co-senior authors.

'Department of Pathology \& Immunology, Baylor College of Medicine and Texas Children's Hospital; 'Department of Pediatrics, Baylor College of Medicine and Texas Children's Cancer Center and
${ }^{3}$ Division of Pediatric Neurosurgery, Department of Surgery, Baylor College of Medicine and Texas Children's Hospital, Houston, TX, USA

Correspondence: CHOLADDA V. CURRY - ccurry@bcm.edu doi:10.3324/haematol.2021.278936

Received: April 8, 2021.

Accepted: June 16, 2021.

Pre-published: June 24, 2021.

Disclosures: no conflicts of interest to disclose.

Contributions: CYB researched the literature, wrote the manuscript, and constructed the tables/figures; ANM, JEA, AMA, and HLW assisted with reviewing medical and pathological records of patients involved, as well as manuscript editing; KEF and CVC conceived the study, interpreted the data, provided feedback and supervision. All authors contributed to patient care, manuscript editing, and evaluation.

\section{References}

1. Liang X, Greffe B, Cook B, et al. Gray zone lymphomas in pediatric patients. Pediatr Dev Pathol. 2011;14(1):57-63.

2. Oschlies I, Burkhardt B, Salaverria I, et al. Clinical, pathological and genetic features of primary mediastinal large B-cell lymphomas and mediastinal gray zone lymphomas in children. Haematologica. 2011;96(2):262-268.

3. Swerdlow SH, Campo E, Harris NL, et al. (Eds.) WHO Classification of Tumours of Haematopoietic and Lymphoid Tissues, Revised 4th ed.; IARC: Lyon, France, 2017.

4. Aussedat G, Traverse-Glehen A, Stamatoullas A, et al. Composite and sequential lymphoma between classical Hodgkin lymphoma and primary mediastinal lymphoma/diffuse large B-cell lymphoma, a clinico-pathological series of 25 cases. Br J Haematol. 2020;189(2):244-256.

5. Zhou T, Bloomquist MS, Ferguson LS, et al. Pediatric myeloid sarcoma: a single institution clinicopathologic and molecular analysis. Pediatr Hematol Oncol. 2020;37(1):76-89.

6. Li MM, Datto M, Duncavage EJ, et al. Standards and guidelines for the interpretation and reporting of sequence variants in cancer: a joint consensus recommendation of the Association for Molecular Pathology, American Society of Clinical Oncology, and College of American Pathologists. J Mol Diagn. 2017;19(1):4-23.

7. Tiacci E, Döring C, Brune V, et al. Analyzing primary Hodgkin and Reed-Sternberg cells to capture the molecular and cellular pathogenesis of classical Hodgkin lymphoma. Blood. 2012;120(23):46094620.

8. Perwein T, Lackner H, Ebetsberger-Dachs G, et al. Management of children and adolescents with gray zone lymphoma: a case series. Pediatr Blood Cancer. 2020;67(5):e28206.

9. Sarkozy C, Chong L, Takata K, et al. Gene expression profiling of gray zone lymphoma. Blood Adv. 2020;4(11):2523-2535.

10. Sarkozy C, Hung SS, Chavez EA, et al. Mutational landscape of grey zone lymphoma. Blood. 2021;137(13):1765-1776.

11. Quintanilla-Martinez L, de Jong D, de Mascarel A, et al. Gray zones around diffuse large $\mathrm{B}$ cell lymphoma. Conclusions based on the workshop of the XIV meeting of the European Association for Hematopathology and the Society of Hematopathology in Bordeaux, France. J Hematop. 2009;2(4):211-236.

12. Sarkozy C, Molina T, Ghesquières $\mathrm{H}$, et al. Mediastinal gray zone lymphoma: clinico-pathological characteristics and outcomes of 99 patients from the Lymphoma Study Association. Haematologica. 2017;102(1):150-159.

13. Wilson WH, Pittaluga S, Nicolae A, et al. A prospective study of mediastinal gray-zone lymphoma. Blood. 2014;124(10):1563-1569.

14. Joos S, Otaño-Joos MI, Ziegler S, et al. Primary mediastinal (thymic) B-cell lymphoma is characterized by gains of chromosomal material including 9p and amplification of the REL gene. Blood. 1996; 87(4):1571-1578.

15. Kimm LR, deLeeuw RJ, Savage KJ, et al. Frequent occurrence of deletions in primary mediastinal B-cell lymphoma. Genes Chromosomes Cancer. 2007;46(12):1090-1097. 